\title{
Heat transport in 3D anelastic simulations of the internal dynamics of giant planets without cores
}

\author{
Martha Evonuk ${ }^{1}$ and Gary A. Glatzmaier ${ }^{2}$ \\ ${ }^{1}$ Institut für Geophysik, ETH Hoengg, 8093 Zürich, Switzerland \\ email: mevonuk@erdw.ethz.ch \\ ${ }^{2}$ Department of Earth and Planetary Sciences, University of California, Santa Cruz \\ 1156 High Street, Santa Cruz, CA 95064, USA \\ email: glatz@es.ucsc.edu
}

\begin{abstract}
Differential rotation, similar to that seen on our gas giants, is manifested at the surface of three-dimensional (3D) computer simulations of thermal convection in density-stratified rotating planets without solid cores. Below the surface, the flow forms short axially-aligned vortices, generated by fluid expanding as it rises and contracting as it sinks. The convergence of the nonlinear Reynolds stresses resulting from the vorticity generated by fluid flowing through the density stratification maintains the surface banded zonal flow without the classical vortex stretching of Taylor columns. These preliminary simulations demonstrate that large nonconvecting cores are not required to obtain multiple zonal jets at the surface, and show greater convective heat flux towards the poles relative to that seen at the equator. This result could help explain the nearly uniform with latitude thermal emission observed at the surface of Jupiter.
\end{abstract}

Keywords. Planets and satellites: general, Jupiter, Saturn, stars: interiors

\section{The numerical model}

We use the finite volume method, as in Evonuk \& Glatzmaier (2006a), in three dimensions to model thermal convection in a rotating spherical body with central gravity. We use the anelastic approximation (Gough 1969; Glatzmaier \& Gilman 1981; Lipps $1990)$, with $\nabla \cdot(\bar{\rho} \mathbf{u})=0$, where variables with overbars represent background functions that vary with radius. The divergence of velocity, the momentum equation, and the heat equation are as follows:

$$
\begin{gathered}
\nabla \cdot \mathbf{u}=-\frac{u_{r}}{\bar{\rho}} \frac{d \bar{\rho}}{d r} \\
\bar{\rho} \frac{\partial \mathbf{u}}{\partial t}=-\bar{\rho}(\mathbf{u} \cdot \nabla) \mathbf{u}-\rho^{\prime} \overline{\mathbf{g}}-2 \bar{\rho} \mathbf{\Omega} \times \mathbf{u}-\nabla P+\nabla \cdot\left[2 \bar{\rho} \bar{\nu}\left(e_{i j}-\frac{1}{3}(\nabla \cdot \mathbf{u}) \delta_{i j}\right)\right] \\
\bar{\rho} \bar{T} \frac{\partial S}{\partial t}=-\bar{\rho} \bar{T}(\mathbf{u} \cdot \nabla) S+\nabla \cdot\left(C_{p} \bar{\rho} \bar{\kappa} \nabla S^{\prime}\right)+\bar{\rho} Q
\end{gathered}
$$

where $\rho$ is the density, $\mathbf{u}$ the velocity, $\mathbf{g}$ the gravity, $\boldsymbol{\Omega}$ the rotation vector, $P$ the pressure, $\nu$ the kinematic viscosity, $T$ the temperature, $S$ the specific entropy, $C_{p}$ the specific heat capacity, $\kappa$ the thermal diffusivity, $e_{i j}$ the rate of strain tensor, and $Q$ a specified internal heating rate.

The dimensionless parameters for our simulation are the Ekman number $(E k=$ $\left.\nu /\left(2 \Omega D^{2}\right)=1.5 \times 10^{-7}\right)$, the Prandtl Number $(\operatorname{Pr}=\nu / \kappa=0.1)$, and the Rayleigh number $\left(R a=g_{o} \Delta S D^{3} /\left(C_{p} \nu \kappa\right)=2.5 \times 10^{10}\right)$, where $\mathrm{D}$ is the depth of the convection 


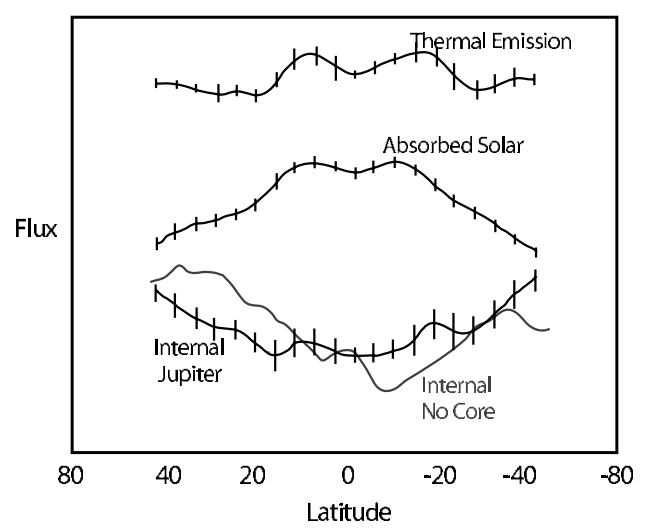

Figure 1. Energy Balance of Jupiter. The solid black lines are the thermal and absorbed energy, and the internal energy is calculated as the excess of the thermal over the absorbed energy (Parraglia 1984). Overlaid, as a grey line, is the internal heat flux for the model simulation with no core.

zone, $g_{o}$ the gravity at the outer boundary, and $\Delta S$ is the drop in the entropy perturbation across the convection zone.

We set the outer boundaries of the simulation to be impermeable and stress free at a constant entropy perturbation of zero. We neglect the magnetic fields but include five density scale heights through the interior of our coreless planet so that the surface of the simulation corresponds to a pressure of $10^{8} \mathrm{~Pa}$ and a density of $2.89 \times 10^{-2} \mathrm{gm} / \mathrm{cm}^{3}$ as in one dimensional models of Jupiter by Guillot (1999). The background temperature gradient is adiabatic and convection is driven by an axially symmetric heating term which tapers to zero by $35 \%$ of the sphere's radius. The rotation rate is about 10 hours, similar to that of Jupiter and Saturn and the model resolution is $400^{3}$ in Cartesian coordinates.

\section{Summary of results}

Our 3D simulation without a core maintains a multiple jet zonal flow structure with a strong equatorial prograde jet. The coupling of the fluid flow with the Coriolis force via the expansion and contraction of fluid as it moves through the density-stratification allows for the local convergence of Reynolds stresses and establishment of differential fluid flow independent of the curved outer boundary, unlike the constant density case which relies on boundary interactions to establish zonal flow structures. For a more extensive discussion of this local mechanism see Glatzmaier, Evonuk \& Rogers (in prep.) and Evonuk \& Glatzmaier (2006b).

Axially aligned vorticity features are observed throughout the simulation. While they do not span the convective zone, they do allow for preferential flow of high entropy along the vorticity features. This preferred transport may be important for giant planets. While strong convection is likely restricted to a thin shell due to suppression of convection in the metallic hydrogen region by the strong magnetic field, the base of this fast convection shell is likely to have non-uniform entropy and heat flux.

Simulation results show to first order lower heat flux in the equatorial regions (Figure 1). The absolute amplitudes of the flux for Jupiter and the simulation are not shown as the simulation is driven much harder than Jupiter to compensate for the necessarily larger diffusivities used in the simulation. Also to first order we see asymmetrical 


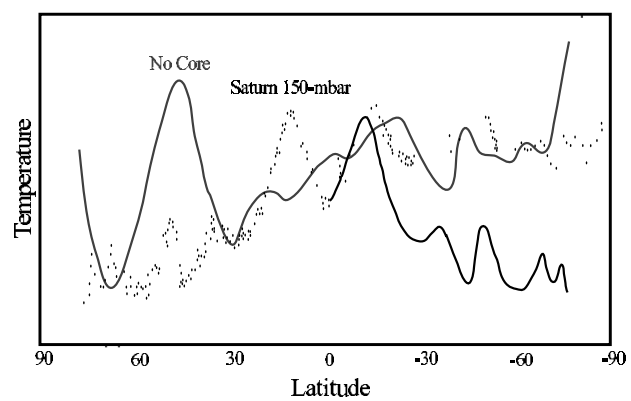

Figure 2. Temperatures retrieved by inversion of Voyager infrared spectral measurements. The broken curve is a fit to the northern hemisphere 150-mbar temperatures which has been folded over at the equator to compare with the southern hemisphere (Conrath and Pirraglia 1983). Overlaid is a similar profile in grey for the simulation with no core.

distributions of the heat at the surface of the planet with an overall trend towards higher temperatures in the southern hemisphere similar to the trend seen at the 150-mbar level in Saturns atmosphere (Figure 2). These results are time dependent and the simulation is not specific to either planet.

These results indicate that a constant entropy, temperature, or heat flux at the base of the convective zone in giant planet simulations may not provide the best lower boundary condition for realistic numerical simulations. Also we see that the density-stratification plays an important role in vorticity generation and the maintenance of a zonal flow structure. It is a local mechanism and is likely to be important in giant planets that are highly turbulent.

\section{Acknowledgements}

Support for this research has been provided by the NASA Outer Planets Research program (NNG05GG69G). Computational resources were provided by NASA (Ames Research Center), NSF (Pittsburgh Supercomputing Center) and DOE (NERSC).

\section{References}

Conrath, B.J. \& Pirraglia, J.A. 1983, Icarus 53, 286

Evonuk, M. \& Glatzmaier, G.A. 2006a, Icarus 181, 458

Evonuk, M. \& Glatzmaier, G.A. 2006b, Proceedings IAU Symposium 239

Glatzmaier, G.A., Evonuk, M. \& Rogers, T.M. in prep.

Glatzmaier, G.A. \& Gilman, P.A. 1981, ApJS 45, 335

Gough, D.O. 1969, J. Atmos. Sci. 26, 448

Guillot, T. 1999, Science 286, 72

Lipps, F.B. 1990, J. Atmos. Sci. 47, 1794

Pirraglia, J.A. 1984, Icarus 59, 169 